\title{
Supervaluationism and Classical Logic
}

\author{
Pablo Cobreros \\ pcobreros@unav.es
}

May 9, 2009

\begin{abstract}
The supervaluationist theory of vagueness provides a notion of logical consequence that is akin to classical consequence. In the absence of a definitely operator, supervaluationist consequence coincides with classical consequence. In the presence of 'definitely', we might find counterexamples to classically valid patterns of inference within supervaluationist reasoning. Foes of supervaluationism emphasize the last result to argue against the supervaluationist theory, concluding that 'supervaluations invalidate our natural mode of deductive thinking' (Williamson, 1994, 152). This and related objections, however, do not consider particular ways in which we might provide systems of deduction for supervaluationist consequence. The present talk considers two ways in which we can carry out this task.
\end{abstract}

\section{Tableaux}

Supervaluationist semantics for a propositional language containing a definitely operator (' $\mathcal{D}$ ' henceforth) can be represented as a possible worlds semantics for a propositional language with a necessity operator (points in a structure are informally read as precisifications instead of worlds and the relation between points is informally read as an admissibility relation instead of an accessibility relation). The standard notion of logical consequence for modal logic is local validity (preservation of truth in each point in every structure); which, in the present context, is considered as classical consequence. Supervaluationism is supposed to be committed to a stronger notion of consequence that preserves the property of truth-in-all-points, in every structure; this is global validity. This section shows how to extend to global validity the standard system of tableaux for local validity (as presented, for example, in Priest (2001)). 
Let $\mathcal{D}_{u}$ be an operator defined as follows (over a propositional language with 'definitely') $: \mathcal{D}_{u} \varphi$ is true at a precisification $p$ in a given interpretation iff $\varphi$ is true in every precisification in the given interpretation. We might establish the following connection between supervaluationist's global validity $\left(\vDash_{g}\right)$ and standard local validity $\left(\vDash_{l}\right)$ :

Claim 1: $\Gamma \vDash_{g} \alpha$ iff $\mathcal{D}_{u}(\Gamma) \vDash_{l} \alpha$ (where $\mathcal{D}_{u}(\Gamma)$ is the result of applying $\mathcal{D}_{u}$ to each $\gamma$ in $\Gamma$ )

We might use this connection to provide a system of tableaux for global validity: $\Gamma \vdash_{g} \alpha$, just in case there is a closed tableau for the set $\mathcal{D}_{u}(\Gamma) \cup$ $\{\neg \alpha\}$.

\section{A more general approach}

A second, more general approach exploits a connection between global and local validity without making use of symbols not already present in the language (such as $\mathcal{D}_{u}$ ). Take a propositional language with ' $\mathcal{D}$ '. The following holds:

Claim 2: $\Gamma \vDash_{g} \varphi$ iff $\left\{\mathcal{D}^{n} \gamma \mid \gamma \in \Gamma, n \in \omega\right\} \vDash_{l} \varphi$

Again we might use this connection to provide suitable proof theory for global validity. Since local validity is standard we might rely on the existence of suitable proof theory for this notion. Take any notion of deduction adequate for local validity, $\vdash_{l}$, and add the following rule:

$\mathcal{D}$-introduction: From $\Gamma \vdash \varphi$ infer $\Gamma \vdash \mathcal{D} \varphi$

The addition of this rule provides a new notion of deductive consequence, $\vdash_{g}$. We can use Claim 2 to show that $\vdash_{g}$ is complete with respect to the global notion of consequence:

Claim 3 If $\Gamma \vDash_{g} \varphi$ then $\Gamma \vdash_{g} \varphi$

The addition of $\mathcal{D}$-introduction to some particular systems requires restricting the applicability of some rules (in order to guarantee soundness). But the proof of Claim 3 shows precisely how these rules should be restricted.

\section{Aim of the talk}

Once we introduce a $\mathcal{D}$ operator, global validity leads to counterexamples to classically valid patterns of inference. This result can be used against the supervaluationist theory in different ways. On the one hand, it might cast doubts on whether it is possible to adapt standard systems of deduction for global validity in a simple way (doubts in this line are expressed in Varzi 
(2007)). On the second hand, it seems to show that supervaluationism cannot invoke its proximity to classical logic as an argument for the theory. The talk addresses directly the first, more technical point, of providing suitable systems of deduction for global validity based on standard ways of deduction. But the talk also brings light on the second, more philosophical, problem since it shows that classically valid ways of reasoning can still be used for global validity placing restrictions on their applicability.

\section{References}

Fine, K. (1975). Vagueness, truth and logic. Synthese, 30:265-300.

Keefe, R. (2000a). Supervaluationism and validity. Philosophical Topics, 28(1):93-105.

Keefe, R. (2000b). Theories of Vagueness. Cambridge University Press.

Priest, G. (2001). An Introduction to Non-Classical Logic. Cambridge Univerisity Press.

Varzi, A. (2007). Supervaluationism and its logic. Mind, 116(463):633-676.

Williams, J. R. G. (2008). Supervaluationism and logical revisionism. The Journal of Philosophy.

Williamson, T. (1994). Vagueness. Routledge. 